Pacific Journal of Mathematics

THE CLASSIFICATION OF CERTAIN CLASSES OF TORSION
FREE ABELIAN GROUPS 


\section{THE CLASSIFICATION OF CERTAIN CLASSES OF TORSION FREE ABELIAN GROUPS}

\section{E. Murley}

Let $\mathscr{A}$ denote the class of torsion free Abelian groups of finite rank. It is shown that for $A \in \mathscr{A}$, there is a quotient divisible subgroup $Q D(A)$ such that $A / Q D(A)$ is a reduced torsion group. Furthermore, $Q D(A)$ and $A / Q D(A)$ are unique up to quasi-isomorphism. Let $\mathscr{B}$ denote the subclass of $\mathscr{A}$ of groups $A$ such that for almost all primes $p$, the $p$-primary component of $A / Q D(A)$ is the direct sum of $r_{p}(A)$ isomorphic cyclic groups where $r_{p}(A)$ denotes the $p$-rank of $A$. The groups in $\mathscr{B}$ are classified up to quasi-isomorphism, which generalizes the Beaumont-Pierce classification of quotient divisible groups.

The main results of this paper concern the subclass $\mathscr{E}$ of $\mathscr{A}$ of groups $A$ such that $r_{p}(A) \leqq 1$ for all primes $p$. The class $\mathscr{E}$ may be profitably treated as a generalization of the class of rank one groups in $\mathscr{A}$.

In $\S 4 \mathscr{E}$ is characterized as a certain subclass of the class of groups in $\mathscr{A}$ whose isomorphism and quasi-isomorphism classes coincide and the groups in $\mathscr{E}$ are classified up to isomorphism. This generalizes the well-known Baer classification of rank one groups in $\mathscr{A}$ and is related to a question of L. Fuchs concerning the structure of torsion free Abelian groups which have hereditary generating systems. In $\S 5$ the endomorphisms of groups in $\mathscr{E}$ are studied. It is shown that every endomorphism of an indecomposable group in $\mathscr{E}$ is an integral multiple of an automorphism. The special groups of $F$. Richman play much the same role in $\mathscr{E}$ that the groups of non-nil type play in the class of rank one groups in $\mathscr{A}$. For example, an indecomposable group $A$ in $\mathscr{E}$ is the additive subgroup of the endomorphism ring of some group in $\mathscr{E}$ if and only if $A$ is a special group.

In the following, $\Pi$ denotes the set of primes in the ring of integers $Z, Z_{p}$ the local subring of the field of rationals $Q$ determined by the prime $p$ and $Z(n)$ the cyclic group of order $n$. The ring of $p$-adic integers and the field of $p$-adic numbers are denoted by $Z^{(p)}$ and $Q^{(p)}$ respectively. Let $M$ be a torsion free module over an integral domain $R$ with quotient field $Q(R)$. Then the rank of $M$, denoted by $r_{R}(M)$, is the $Q(R)$-dimension of $Q(R) \otimes_{R} M$. If $R=Z$, then we let $r(M)=r_{Z}(M)$ and call a subgroup $N$ full in $M$ if $r(M)=$ $r(N)$. If $B$ is a $p$-primary abelian group and $B[p]=\{x \in B \mid p x=0\}$, then the rank of $B$, denoted by $r(B)$, is the $Z(p)$-dimension of $B[p]$. The p-rank of a torsion free group $B$, denoted by $r_{p}(A)$, is $r(B / p B)$. 
Let $B$ and $C$ be Abelian groups. Then $B \otimes C$ and $\operatorname{Hom}(B, C)$ will mean $B \otimes_{Z} C$ and $\operatorname{Hom}_{Z}(B, C)$. The endomorphism ring of $B$ is denoted by $\operatorname{End}(B)$. We let $B_{p}$ denote the $p$-primary component of $B, d(B)$ be the maximal divisible subgroup of $B$ and for $B$ torsion free, $\Pi(B)=\{p \in \Pi \mid p B=B\}=\left\{p \in \Pi \mid r_{p}(B)=0\right\}$. If $H$ is a characteristic, then the type determined by $H$ is denoted by $[H]$. Let $B$ be torsion free and $x \in B$. Then the height of $x$ in $B$ is a characteristic which we denote by $H^{B}(x)$. The inner type of $B$ [13], denoted by $\tau_{*}(B)$, is the greatest lower bound in the lattice of types of the type set of $B$, i.e. $\left\{\left[H^{B}(x)\right] \mid 0 \neq x \in B\right\}$. For $H$ a characteristic, we let $B[H]=\left\{x \in B \mid H^{B}(x) \geqq H\right\}$, which is a fully invariant subgroup of $B$. Epimorphisms [monomorphisms] are denoted by $\rightarrow[\mapsto]$.

Much of this paper is contained in the author's doctoral thesis supervised by Professor R. A. Beaumont at the University of Washington, Seattle, Washington. The author is also indebted to Professor R. B. Warfield, Jr., for several valuable suggestions concerning the endomorphism rings of groups in $\mathscr{E}$ and to the referee for suggesting many substantial improvements.

1. The local-global setting. Throughout $A$ will denote a torsion free abelian group of finite rank and will be considered as a full subgroup of a fixed finite dimensional rational vector space $V$. Any group in $\mathscr{A}$ with the same rank as $A$ can be imbedded in $V$ as a full subgroup and $V \cong Q \otimes A$. Let $V^{(p)}=Q^{(p)} \otimes V$ for $p \in \Pi$. Then we regard the $Q^{(p)}$-module $V^{(p)}$ as an extension of $V$ where $V^{(p)} \cap$ $V^{(p)}=V$ for $p \neq q$. Let $B$ be a subgroup of $A$. Then $Z_{p} B$ denotes the $Z_{p}$-submodule of $V$ generated by $B$ and $B^{(p)}$ denotes the $Z^{(p)}$-submodule of $V^{(p)}$ generated by $B$. Since $V^{(p)}$ is viewed as an extension of $V$ and torsion free groups are flat, there is a natural identification between $A^{(p)}$ and $Z^{(p)} \otimes A$ given by $\Sigma r_{i} a_{i} \rightarrow \Sigma r_{i} \otimes a_{i}$ for $r_{i} \in Z^{(p)}$ and $a_{i} \in A$. A similar identification occurs between $Z_{p} A$ and $Z_{p} \otimes A$. Note that $r(A)=r_{Z^{(p)}}\left(A^{(p)}\right)=r_{Q^{(p)}}\left(V^{(p)}\right)=r_{Z_{p}}\left(Z_{p} A\right)$. The following wellknown local-global relations will be frequently used:

(i ) $A^{(p)} \cap V=Z_{p} A$

(ii) $\bigcap_{q \in \Pi} Z_{p} A=A=\bigcap_{q \in \Pi} A^{(p)}$

(iii) $p^{n} Z_{p} A \cap A=p^{n} A=p^{n} A^{(p)} \cap A$ for $n \geqq 0$

(iv) for $B$ a full subgroup of $A, Z_{p} A / Z_{p} B \cong(A / B)_{p} \cong A^{(p)} / B^{(p)}$.

Two groups $B$ and $C$ are quasi-isomorphic, denoted by $B \cong C$, if there are subgroups $B^{\prime} \subseteq B$ and $C^{\prime} \subseteq C$ such that $B^{\prime} \cong C^{\prime}$ and $B / B^{\prime}$, $C / C^{\prime}$ are groups of bounded order. For $B$ and $C$ torsion, $B \cong C$ if and only if $B_{p} \doteq C_{p}$ for all $p$ and $B_{p} \cong C_{p}$ for almost all $p$ [4]. Thus, if $B$ and $C$ are torsion homomorphic images of $A$, then $B \cong C$ if and only if $d(B) \cong d(C)$ and $B_{p} \cong C_{p}$ for almost all $p$. Let $B$ and 
$C$ be torsion free. Then $B \doteq C$ is equivalent to the existence of a monomorphism $\phi$ on $B$ into $C$ such that $C / \phi(B)$ is of bounded order. It is well-known that if $B \cong A$ and $B \cong A$, then $A / B$ is a finite group. This has the important consequence that $A \doteq B$ if and only if each group is imbeddable in the other one. $B$ and $C$ are quasiequal, denoted by $B \doteq C$, if there are positive integers $n$ and $m$ such that $B \supseteqq n C \supseteqq m B$. Two torsion free $Z_{p}$ modules are quasi-equal if they are quasi-equal as groups. The local-global relations give: $A \doteq B$ if and only if $Z_{p} A=Z_{p} B$ for almost all $p$ and $Z_{p} A \doteq Z_{p} B$ for all $p$.

Lemma 1. Let $B$ and $C$ be full subgroups of $A$. If $B \doteq C$, then $A / B \cong A / C$.

Proof. Since $Z_{p} B=Z_{p} C$ for almost all $p,(A / B)_{p} \cong Z_{p} A / Z_{p} B=$ $Z_{p} A / Z_{p} C \cong(A / C)_{p}$ for almost all $p$. Now $C \supseteqq m B \supseteqq n C$ for some $n, m>0$. Let $B^{\prime}=m B$. Since $C / B^{\prime}$ is bounded, the exact sequence $C / B^{\prime} \stackrel{i d}{\longrightarrow} A / B^{\prime} \rightarrow A / C$ shows that $d\left(A / B^{\prime}\right) \cong d(A / C)$. Since $A$ is torsion free, $A / B \cong m A / m B=m\left(A / B^{\prime}\right)$ and so $d(A / B) \cong d\left(A / B^{\prime}\right)$. Hence, $A / B \doteq A / C$.

Definition. $k_{p}(A)=r\left(d_{p}(A / I)\right)$ where $I$ is a full, free subgroup of $A$ and $s_{p}(A)=r\left(\bigcap_{n} p^{n} A\right)$.

Note that $k_{p}(A)$ does not depend upon $I$ by Lemma 1 . As in [1] we let $\delta_{p}(A)$ denote the maximal divisible subgroup of $A^{(p)}$, which is the maximal divisible submodule of $A^{(p)}$ regarded as a $Z^{(p)}$ module. Thus, $\delta_{p}(A)$ is a $Q^{(p)}$ subspace of $V^{(p)}$.

Lemma 2. (i) $r(A)=r_{p}(A)+k_{p}(A)$

(ii) $k_{p}(A)=r_{Z^{(p)}}\left(\delta_{p}(A)\right)$

(iii) $s_{p}(A)=r\left(\delta_{p}(A) \cap V\right)$

Proof. For (i), let $I$ be a full, free subgroup of $A$ such that $(A / I)_{p}$ is divisible. Then $(V / I)_{p} \cong(A / I)_{p} \oplus(V / A)_{p}$ and so $r(V)=$ $k_{p}(A)+r\left((V / A)_{p}\right)$. Since $V / A \cong V / p A$ and $V / p A[p]=A / p A, r\left((V / A)_{p}\right)=$ $r(A / p A)$, which gives (i). To show (ii) it will be enough in view of (i ) to show $r(A)=r_{p}(A)+r_{Z^{(p)}}\left(\delta_{p}(A)\right)$. Now $Z^{(p)} \otimes A=d\left(Z^{(p)} \otimes A\right) \oplus F$ where $F$ is a free $Z^{(p)}$-module [5, 44.2] and so $r(F / p F)=r_{Z}(p)(F)$. Thus, it will be sufficient to show $r_{p}(A)=r(F / p F)$. The exact sequence $\quad p A \stackrel{i d}{\longrightarrow} A \rightarrow A / p A \quad$ implies $\quad Z^{(p)} \otimes p A>\stackrel{e}{\longrightarrow} Z^{(p)} \otimes A \rightarrow$ $Z^{(p)} \otimes(A / p A)$ exact. Note that $e\left(Z^{(p)} \otimes p A\right)=p\left(Z^{(p)} \otimes A\right)$. Thus $F / p F \cong Z^{(p)} \otimes A / p\left(Z^{(p)} \otimes A\right) \cong Z^{(p)} \otimes(A / p A) \cong A / p A$, which gives (ii). For (iii), note that $Z_{p}\left(\bigcap_{n} p^{n} A\right)=d\left(Z_{p} A\right)=\delta_{p}(A) \cap V$.

Corollary 1. Let $B$ be a full subgroup of $A$. Then the following 
conditions are equivalent: (i) $(A / B)_{p}$ is reduced, (ii) $\delta_{p}(A)=\delta_{p}(B)$, (iii) $k_{p}(A)=k_{p}(B)$, and (iv) $r_{p}(A)=r_{p}(B)$.

Lemma 3. Let $B$ be a subgroup of $A$ and $H$ a characteristic such that $\{p \in \Pi \mid H(p)=\infty\}=\Pi(A)$. Then the following are equivalent:

(i) $B=A[H]$ and $\tau_{*}(A) \geqq[H]$

(ii) $A / B$ is torsion with $(A / B)_{p} \cong \bigoplus^{r_{p}(A)} Z\left(p^{H(p)}\right)$ for $p \notin \Pi(A)$ and $(A / B)_{p}=\{0\}$ for $p \in \Pi(A)$

(iii) $p^{H(p)} Z_{p} A=Z_{p} B$ for $H(p)<\infty$ and $Z_{p} A=Z_{p} B$ for $H(p)=\infty$.

Proof. We give a cyclical proof. Assume (i ). Then $\tau_{*}(A) \geqq$ $[H]$ gives $A / B$ torsion. Since $A[H]=\bigcap_{q_{\Pi \Pi(A)}} q^{H(q)} A, Z_{p} B=Z_{p}\left(p^{H(p)} A\right)$ for $p \notin \Pi(A)$ and $Z_{p} B=Z_{p} A$ for $p \in \Pi(A)$. Thus, $(A / B)_{p}=\{0\}$ for $p \in \Pi(A)$ and $(A / B)_{p} \cong A / p^{H(p)} A \cong \bigoplus^{r_{p}(A)} Z\left(p^{H(p)}\right)$ for $p \notin \Pi(A)$, which is (ii). Assume (ii). Then $Z_{p} A=Z_{p} B$ for $p \in \Pi(A)$. For $p \notin \Pi(A)$, $p^{H(p)} Z_{p} A \subseteq Z_{p} B \subseteq Z_{p} A$ and $Z_{p} A / p^{I I(p)} Z_{p} A$ is a $p$-group, with the same order as $Z_{p} A / Z_{p} B$. Thus, $p^{H(p)} Z_{p} A=Z_{p} B$ for $p \notin \Pi(A)$, which is (iii). Assume (iii). Since $A / A[H]$ is torsion, $\tau^{*}(A) \geqq[H]$. Since $\Pi(A)=\Pi(B), B=$ $\bigcap_{H(p)<\infty}\left(p^{H(p)} Z_{p} A \cap V\right)=\bigcap_{H(p)<\infty}\left(p^{H(p)} Z_{p} A \cap A\right)=\bigcap_{H(p)<\infty} p^{H(p)} A=A[H]$, which is (i).

Corollary 2. If $\tau_{*}(A) \geqq[H]$, then End $(A) \cong$ End $(A[H])$.

Proof. For $\phi \in$ End $(A)$, let $\phi^{\prime}$ be the restriction of $\phi$ to $A[H]$. Since $A[H]$ is a full, fully invariant subgroup of $A, \phi \rightarrow \phi^{\prime}$ is a ring monomorphism into End $(A[H])$. For $\lambda^{\prime} \in$ End $(A[H])$, let $\lambda$ be its unique extension to $A$ into $V$. By Lemma $3, p^{H(p)} Z_{p} A=Z_{p} A[H] \supseteqq$ $Z_{p}(\lambda(A[H]))=p^{H(p)} Z_{p} \lambda(A)$ for $p \notin \Pi(A)$. Thus, $Z_{p} \lambda(A) \subseteq Z_{p} A$ for all $p$ and so $\lambda \in$ End $(A)$.

2. The quotient divisible core. We recall from [1] that $A$ is a quotient divisible $[Q D]$ group if $A$ has a full free subgroup $I$ such that $A / I$ is divisible. Note that $A$ is a $Q D$ group if and only if for $J$ a full free subgroup of $A, A / J=D \oplus T$ where $D$ is divisible and $T$ is finite. The invariants introduced by Beaumont-Pierce in [1] to classify the $Q D$ groups in $\mathscr{A}$ involve the following considerations. Let $\mathscr{L}_{p}(V)$ denote the lattice of all $Q^{(p)}$-subspaces of $V^{(p)}$ and $\mathscr{L}^{\prime}(V)=$ $\Pi_{p} \mathscr{L}_{p}(V)$ the direct product of these lattices. If $\delta \in \mathscr{L}(V)$, then the $p$-component of $\delta$ is denoted by $\delta_{p}$ and $\delta$ is referred to as a $Q D i n$ variant (associated with $V$ ). For $\phi$ a $Q$-automorphism of $V$, let $\phi^{(p)}=$ $\dot{\phi} \otimes i d_{Q^{(p)}}$, which is a $Q^{(p)}$-automorphism of $V^{(p)}$.

Definition. Let $\delta, \delta^{\prime} \in \mathscr{L}(V)$. Then $\delta \leqq \delta^{\prime}$ if there is a $Q$-automorphism $\phi$ of $V$ such that $\phi^{(p)}\left(\delta_{p}\right) \subseteq \delta_{p}^{\prime}$ for all $p$. $\delta \sim \delta^{\prime}$ if $\delta \leqq \delta^{\prime}$ and $\delta^{\prime} \leqq \delta$. For $A$ full in $V$, let $\delta(A) \in \mathscr{L}(V)$ such that $\delta(A)_{p}=\delta_{p}(A)$. 
Let $A$ and $B$ be full $Q D$ subgroups of $V$. Then the BeaumontPierce $Q D$ Theorem $[1,5.25]$ states that:

(i) $A$ is imbeddable in $B$ if and only if $\delta(A) \leqq \delta(B)$,

(ii) $A \doteq B[A \doteq B]$ if and only if $\delta(A)=\delta(B)[\delta(A) \sim \delta(B)]$,

(iii) For $\delta \in \mathscr{L}(V)$, there is a full $Q D$ subgroup $A$ of $V$ such that $\delta(A)=\delta$.

Definition. Let $I$ be a full free subgroup of $A$ and $\phi$ be the natural map $A \rightarrow A / I$. Then $Q D(A, I)=\phi^{-1}(d(A / I))$.

Lemma 4. Let $I$ and $J$ be full free subgroups of $A$ and let $B$ be a full subgroup of $A$. Then:

(i) $B$ contains $I$ and $\delta(A)=\delta(B)$ if and only if $Q D(A, I) \cong B$

(ii) if $B$ is $Q D$ and $\delta(A)=\delta(B)$, then $Q D(A, J)=B$ for some $J$

(iii) $Q D(A, I) \doteq Q D(A, J)$ and $A / Q D(A, I) \doteq A / Q D(A, J)$.

Proof. First note that $Q D(A, I)$ is a full $Q D$ subgroup of $A$ such that $A / Q D(A, I)$ is reduced torsion. Part ( $\mathrm{i}$ ) is now immediate from Corollary 1. For (ii), let $J$ be a full free subgroup of $B$ such that $B / J$ is divisible. The first part gives $Q D(A, J) \subseteq B$ and so $B / J \cong$ $Q D(A, J) / J \oplus B / Q D(A, J)$, which shows that $B / Q D(A, J)$ is divisible. Since $\delta(B)=\delta(Q D(A, J)), B / Q D(A, J)$ is reduced by Corollary 1 . Hence, $B=Q D(A, J)$, which is (ii). For the first part of (iii), you may invoke the Beaumont-Pierce $Q D$ Theorem or more directly, note that $Q D(A, I)+J$ is a $Q D$ subgroup of $A$ which is quasi-equal to $Q D(A, I)$. Since $Q D(A, I)+J \supseteqq Q D(A, I+J) \supseteqq Q D(A, I)$ by (i), $Q D(A, I) \doteq Q D(A, I+J)$. Thus, $Q D(A, I) \doteq Q D(A, J)$ by symmetry. The second part of (iii) is now immediate from Lemma 1.

For the remainder of this section $I$ will denote a full free subgroup of $A$. Note that $A$ is a locally free group, i.e. $Z_{p} A$ is a free $Z_{p}$ module for all $p$, if and only if $Q D(A, I)=I$ and $A$ is a $Q D$ group if and only if $Q D(A, I) \doteq A$. The quasi-isomorphism class determined by $Q D(A, I)$, which by Lemma 4 is independent of the choice of $I$, will be referred to as the $Q D$ core of $A$. The Beaumont-Pierce $Q D$ Theorem shows that two groups $A$ and $B$ have the same $Q D$ core if and only if $\delta(A) \sim \delta(B)$. The quasi-isomorphism class determined by $A / Q D(A, I)$, which by Lemma 4 is independent of choice of $I$, is closely related to the Richman type of $A$. See [12] or [13].

Let $A / I=d(A / I) \oplus T$. Then $T \cong A / Q D(A, I)$ and $r\left(T_{p}\right) \leqq r_{p}(A)$ for all $p$ by Lemma 2. Thus, for $r_{p}(A)>0,(A / Q D(A, I))_{p}=\bigoplus_{i=1}^{r_{p}^{p}(A)} Z\left(p^{\alpha_{i}(p)}\right)$ where $0 \leqq \alpha_{i}(p) \leqq \alpha_{j}(p)<\infty$ for $j>i$ and $(A / Q D(A, I))_{p}=\{0\}$ for $r_{p}(A)=0$. 
Definition. For $p \in \Pi$, let $H_{*}(A, I)(p)=\alpha_{1}(p)$ if $r_{p}(A)>0$ and $\infty$ otherwise, $H^{*}(A, I)(p)=\alpha_{s}(p)$ where $s=r_{p}(A)$ if $r_{p}(A)>0$ and $\infty$ otherwise, and $\tau^{*}(A)=\left[H^{*}(A, I)\right]$.

Lemma 4 shows that the types $\tau^{*}(A)$ and $\left[H_{*}(A, I)\right]$ are independent of the choice of $I$. The identification of $\left[H_{*}(A, I)\right]$ in (i) of the following was also noted by Warfield [13, p. 194].

\section{LEMMA 5. (i ) $\tau_{*}(A)=\left[H_{*}(A, I)\right]$}

(ii) $\tau_{*}(A)=\tau_{*}(B)$ and $\tau^{*}(A)=\tau^{*}(B)$ whenever $A \doteq B$ (iii) $A$ is a $Q D$ group if and only if $\tau^{*}(A)$ is non-nil.

Proof. For (i), let $H \in \tau_{*}(A)$ and $I$ be a full free subgroup of $A[H]$. To see that $H_{*}(A, I) \geqq H$, refer to Lemmas 3 and 4 to note that $Q D(A, I) \leqq A[H]$ and consider the orders of the finite $p$-groups $(A / Q D(A, I))_{p},(A / A[H])_{p}$ and the natural map $A / Q D(A, I) \rightarrow A / A[H]$. On the other hand, let $B$ be a group such that $Q D(A, I) \subseteq B \subseteq A$ and for $p \notin \Pi(A),(A / B)_{p} \cong \bigoplus^{r_{p}^{(A)}} Z\left(p^{\alpha}\right)$ where $\alpha=H_{*}(A, I)(p)$. Then $\tau_{*}(A) \geqq\left[H_{*}(A, I)\right]$ by Lemma 3 , which gives (i). Finally, (ii) is an easy computation while (iii) is immediate from the definitions.

We have shown that every full subgroup $A$ of $V$ is an extension of a $Q D$ group $B$ by a reduced torsion group $C$ and that $B$ and $C$ are unique up to quasi-isomorphism. On the other hand, let $B$ be a full $Q D$ group in $V$ and $C$ be a reduced torsion group such that $r_{p}(B) \geqq$ $r\left(C_{p}\right)$ for all $p$. Then there is a full subgroup $A$ in $V$ which is an extension of $B$ by $C$. This may be seen by observing that $r_{p}(B)=$ $r\left((V / B)_{p}\right)$ for all $p$ and letting $A$ be the inverse image in $V$ of an appropriate subgroup of the divisible group $V / B$. Now suppose both $A$ and $A^{\prime}$ are extensions of $B$ by $C$. Then it is easily seen that $A$ and $A^{\prime}$ have the same $Q D$ core, $\tau^{*}(A)=\tau^{*}\left(A^{\prime}\right)$ and $\tau_{*}(A)=\tau_{*}\left(A^{\prime}\right)$. Thus, $A \cong A^{\prime}$ whenever $r(B)=1$. In the next section we study a class of groups $A$ which are determined up to quasi-isomorphism by $B$ and $C$. In contrast we give the following example of two nonquasi-isomorphic groups $A$ and $A^{\prime}$ with the same $Q D$ core and $A / Q D(A, I) \doteq A^{\prime} / Q D\left(A^{\prime}, J\right)$.

ExAMPle 1. Let $A$ and $A^{\prime}$ be locally free, completely decomposable groups of rank 2 whose type sets, denoted by $T(-)$, satisfy $T(A) \neq T\left(A^{\prime}\right), \sup T(A)=\sup T\left(A^{\prime}\right)$, and $\tau_{*}(A)=\inf T(A)=\inf T\left(A^{\prime}\right)=$ $\tau_{*}\left(A^{\prime}\right)$. Such pairs of groups exist in abundance. Let $I$ and $J$ be full free subgroups of $A$ and $A^{\prime}$ respectively. Then $Q D(A, I)=I$ and $Q D\left(A^{\prime}, J\right)=J$ (since the groups are locally free). Thus, $Q D(A, I) \doteq$ $Q D\left(A^{\prime}, J\right)$. A simple computation shows $\tau^{*}(A)=\sup T(A)=\tau^{*}\left(A^{\prime}\right)$. 
Hence, $A / Q D(A, I) \doteq A^{\prime} / Q D\left(A^{\prime}, J\right)$ (since $A$ and $A^{\prime}$ have rank 2) and $A$ is not $\dot{\cong}$ to $A^{\prime}$ (since $T(A) \neq T\left(A^{\prime}\right)$ ).

\section{The Class $\mathscr{B}$.}

Definition. $\mathscr{B}=\left\{A \in \mathscr{A} \mid \tau^{*}(A)=\tau_{*}(A)\right\}$

Note that $\tau^{*}(A)=\tau_{*}(A)$ describes the condition that for $I$ a full free subgroup of $A,(A / Q D(A, I))_{p}$ is a direct sum of $r_{p}(A)$ isomorphic cyclics for almost all $p$. The finite rank $Q D$ groups and the finite rank, homogeneous, completely decomposable groups are examples of groups in $\mathscr{B}$. In fact, the locally free groups in $\mathscr{B}$ are necessarily homogeneous, completely decomposable groups [13, Corollary 5]. Since $\tau^{*}(-)$ and $\tau_{*}(-)$ are quasi-isomorphism invariants [Lemma 5], $A \in \mathscr{B}$ whenever $A \doteq B$ and $B \in \mathscr{B}$. Finally, we mention that $\mathscr{B}$ is closed with respect to direct summands and finite direct sums of the form $\bigoplus_{i=1}^{n} A_{i}$ where $A_{i} \in \mathscr{B}$ and $\tau_{*}\left(A_{i}\right)=\tau_{*}\left(A_{j}\right)$ for all $i, j$.

Lemma 6. Let $H \in \tau_{*}(A)$. Then $A \in \mathscr{B}$ if and only if $Q D(A, J)=$ $A[H]$ for some full free subgroup $J$ in $A$.

Proof. Let $A \in \mathscr{B}$ and let $I$ be a full free in $A[H]$. Then $Q D(A, I) \subseteq A[H]$ by Lemmas 3 and 4 . Since $H \sim H_{*}(A, I)$ by Lemma 5 , Lemma 3 (ii) gives $(A / A[H])_{p} \cong(A / Q D(A, I))_{p}$ for almost all $p$. This says that $A[H] / Q D(A, I)$ is finite. Thus, $A[H]$ is a $Q D$ group such that $A / A[H]$ is reduced torsion. It follows from Lemma 4 that $A[H]=$ $Q D(A, J)$ for some full free $J$ in $A$. The converse is immediate from Lemma 3.

DEFINITION. A type $\tau$ and a $Q D$ invariant $\delta \in \mathscr{L}(V)$ are compatible if the $p^{\text {th }}$-component of $\tau$ is $\infty$ if and only if $\delta_{p}=V^{(p)}$. Note that $\tau_{*}(A)$ and $\delta(A)$ are compatible for $A \in \mathscr{A}$.

THEOREM 1. Let $A$ and $B$ be groups in $\mathscr{B}$ which are full in $V$.

(i) There is an imbedding of $A$ in $B$ if and only if $\delta(A) \leqq \delta(B)$ and $\tau_{*}(A) \leqq \tau_{*}(B)$.

(ii) $A \doteq B$ if and only if $\delta(A)=\delta(B)$ and $\tau_{*}(A)=\tau_{*}(B)$.

(iii) $A \doteq B$ if and only if $\delta(A) \sim \delta(B)$ and $\tau_{*}(A)=\tau_{*}(B)$.

(iv) If $\delta \in \mathscr{L}(V)$ and $\tau$ is a type compatible with $\delta$, then there is a group $C$ in $\mathscr{B}$ which is full in $V$ such that $\delta(C)=\delta$ and $\tau_{*}(C)=$ $\tau$.

Proof of $(i)$. Let $\phi$ be an imbedding of $A$ in $B$ and assume that $\phi$ is extended to a $Q$-automorphism of $V$. Then it is clear that 
$\phi^{(p)}\left(\delta_{p}(A)\right) \subseteq \delta_{p}(B)$ for all $p$, i.e. $\delta(A) \leqq \delta(B)$. Let $I$ be a full free subgroup of $A$. Then $\phi(I)$ is a full free subgroup of $B$ and $A / I \cong$ $\phi(A) / \phi(I) \subseteq B / \phi(I) . \quad$ A modest computation shows $H_{*}(\phi(A), \phi(I)) \leqq$ $H_{*}(B, \phi(I))$, i.e. $\tau_{*}(A) \leqq \tau_{*}(B)$.

Conversely, let $H_{1} \in \tau_{*}(A)$ and $H_{2} \in \tau_{*}(B)$ such that $H_{1} \leqq H_{2}$. Use Lemma 6 to obtain full free subgroups $I$ and $J$ of $A$ and $B$ respectively such that $A\left[H_{1}\right]=Q D(A, I)$ and $B\left[H_{2}\right]=Q D(B, J)$. Since $\delta(A) \leqq$ $\delta(B)$, the Beaumont-Pierce $Q D$ Theorem gives an imbedding $\phi: A\left[H_{1}\right] \succ$ $B\left[H_{2}\right]$. Assume that $\phi$ is uniquely extended to $A$ into $V$ and so for $p \in \Pi(B), Z_{p} \phi(A) \subseteq V=Z_{p} B$. For $p \notin \Pi(B), p^{H_{1}(p)} Z_{p} \phi(A)=Z_{p} \phi\left(A\left[H_{1}\right]\right) \subseteq$ $Z_{p} B\left[H_{2}\right]=p^{H_{2}(p)} Z_{p} B$ by Lemma 3 . Since division is unique in $V, Z_{p} \phi(A) \subseteq p^{t} Z_{p} B \subseteq Z_{p} B$ where $t=H_{2}(p)-H_{1}(p)$. Hence, $\phi(A) \subseteq B$.

Proof of $(i i)$. The "only if" part is immediate. For the converse let $H_{1}=H_{2}$ and $I, J$ be as in the proof of $(i)$. Since $\delta(A)=$ $\delta(B), A\left[H_{1}\right]=Q D(A, I) \doteq Q D(B, J)=B\left[H_{2}\right]$ and so the imbedding $\phi$ in the previous part may be chosen to be a left multiplication by some positive integer $n$. The argument in the previous part now gives $n A \subseteq B$. By symmetry, $m B \subseteq A$ for some $m>0$ and so $A \doteq B$.

Proof of (iii). Since $A \stackrel{\cong}{ } B$ if and only if each group is imbeddable in the other, (iii) is immediate from (i).

Proof of $(i v)$. Let $B$ be a full $Q D$ subgroup of $V$ with $\delta(B)=\delta$. Let $H \in \tau$ and $C$ be a subgroup of $V$ containing $B$ such that $(C / B)_{p} \cong$ $\bigoplus^{r^{(B)}} Z\left(p^{H(p)}\right)$ for $p \notin \Pi(B)$. Note that such a $C$ exists since $V / B$ is divisible with $r\left((V / B)_{p}\right)=r_{p}(B)$. Since $\tau$ and $\delta$ are compatible, $C / B$ is reduced torsion and Lemma 4 (ii) gives $B=Q D(C, I)$ where $I$ is a full free subgroup of $B$ such that $B / I$ is divisible. Thus, $C \in \mathscr{B}$ with $\delta(C)=\delta$ and $\tau_{*}(C)=\tau$.

REMARK 1. Let $A$ be a $Q D$ group and $H$ the characteristic such that $H(p)=\infty$ if $\delta_{p}(A)=V^{(p)}$ and $H(p)=0$ otherwise. Then by Lemma 5 (iii) $[H]=\tau_{*}(A)$. Thus, $\tau_{*}(A)$ may be recaptured from $\delta(A)$ whenever $A$ is a $Q D$ group. This shows that Theorem 1 is a generalization of [1, 5.25]. We mention that the Warfield Duality [13] may be used to show that for $D$ a torsion free, rank 1 group with $\tau_{*}(D)=\tau_{*}(A)$ and $I$ a full free subgroup of $A$, then the following are equivalent: (i ) $A \in \mathscr{B}$, (ii) $Q D(A, I) \doteq \operatorname{Hom}(D, A)$, (iii) $A \doteq D \otimes$ $Q D(A, I)$. This, of course, yields another proof of Theorem 1 .

Lemma 7. If $I$ is a full free subgroup of $A$ and $A \in \mathscr{B}$, then $Q \otimes$ End $(A) \cong Q \otimes$ End $(Q D(A, I))$. 
Proof. Let $H \in \tau_{*}(A)$ and $J$ be a full free subgroup of $A$ such that $A[H]=Q D(A, J)$ [Lemma 6]. Thus, $A[H] \doteq Q D(A, I)$ and so $Q \otimes$ End $(A) \cong Q \otimes$ End $(A[H])=Q \otimes$ End $(Q D(A, I))$ by Corollary 2.

For the following corollary, recall that $A$ is quasi-decomposable if it is quasi-equal to the direct sum of two nonzero torsion free groups. $A$ is strongly indecomposable if it is not quasi-decomposable.

CoRollary 3. Let I be a full free subgroup of $A$ and $A \in \mathscr{B}$. Then the following are equivalent:

(i) $A$ is quasi-decomposable

(ii) $Q D(A, I)$ is quasi-decomposable

(iii) there are nonzero subspaces $U$ and $W$ such that $V=U \oplus W$ and $\delta_{p}(A)=\delta_{p}(A) \cap U^{(p)} \oplus \delta_{p}(A) \cap W^{(p)}$ for all $p$.

Proof. Since a group $B$ in $\mathscr{A}$ is quasi-decomposable if and only if $Q \otimes$ End $(B)$ is decomposable as a module over itself [11], the equivalence of ( $\mathrm{i}$ ) and (ii) is immediate from Lemma 7. (iii) is a necessary and sufficient condition for a $Q D$ group in $\mathscr{A}$ to be quasi-decomposable [1, 5.26]. Since $\delta(A)=\delta(Q D(A, I)$ ), (ii) and (iii) are equivalent. Note that the quotient-divisibility of $A$ must be added to the hypotheses of $[1,5.26]$.

4. The Class $\mathscr{E}$.

Definition. $\mathscr{E}=\left\{A \in \mathscr{A} \mid r_{p}(A) \leqq 1\right.$ for all $\left.p \in \Pi\right\}$.

We note that $\mathscr{E}$ is a subclass of $\mathscr{B}$ which contains the torsion free, rank one groups. Furthermore, the reduced groups $A$ in $\mathscr{E}$ are up to isomorphism precisely the finite rank, pure subgroups of $\prod_{p \in \Pi} Z^{(p)}$, the $Z$-adic completion of the integers. The class $\mathscr{E}$ is closed with respect to pure subgroups, torsion free homomorphic images and tensor products. Here we make use of the facts that for $A, B \in \mathscr{A}$, $r_{p}(A \otimes B)=r_{p}(A) r_{p}(B)$ and for $B$ pure in $A, r_{p}(A)=r_{p}(B)+r_{p}(A / B)$. Recall that a group $A$ in $\mathscr{A}$ is cohesive if $A \in \mathscr{E}$ and $s_{p}(A)=0$ for $p \notin \Pi(A)$ [4]. If $A$ is a non-cohesive group in $\mathscr{E}$, i.e. $0<s_{p}(A)<r(A)$ for some $p$, then $A$ is not homogeneous (since there are $0 \neq x, y \in A$ such that $H^{A}(x)(p)=\infty$ and $\left.H^{A}(y)(p)<\infty\right)$. Thus, the homogeneous groups in $\mathscr{E}$ are cohesive. On the other hand, Theorem 4 in [4] shows the existence of homogeneous and non-homogeneous cohesive groups of any rank greater than one. Richman's special groups [12] are a subclass of the homogeneous groups in $\mathscr{E}$. Reduced groups in $\mathscr{E}$ can be decomposable. For example, if $\left\{\Pi^{\prime}, \Pi^{\prime \prime}\right\}$ is a nontrivial partition of $\Pi, Z\left(\Pi^{\prime}\right)=\bigcap_{p \in \Pi^{\prime}} Z_{p}, Z\left(\Pi^{\prime \prime}\right)=\bigcap_{p \in \Pi^{\prime \prime}} Z_{p}$, and $A=Z\left(\Pi^{\prime}\right) \oplus Z\left(\Pi^{\prime \prime}\right)$, then $A \in \mathscr{E}$. On the other hand, the reduced cohesive groups in $\mathscr{E}$ are 
purely indecomposable, i.e. every pure subgroup is indecomposable [4, C5]. Later we show that $\mathscr{E}$ contains abundant non-cohesive, indecomposable groups which need not be purely indecomposable.

Lemma 8. $A \in \mathscr{E}$ if and only if $A \in \mathscr{A}$ and all finite homomorphic images of $A$ are cyclic.

Proof. If $A \in \mathscr{E}$ and $A / B$ is a group of order $n$, then $A / B$ is the homomorphic image of the cyclic group $A / n A$. Conversely, since $A$ has finite rank, $A / p A$ is a finite group and thus, cyclic.

Definition. $\mathscr{C}=\{A \in \mathscr{A} \mid A \doteq B$ implies $A \cong B\} . \quad \mathscr{D}=\{A \in$ $\mathscr{A} \mid B \cong A$ and $B \cong A$ implies $B=n A$ for some $n\}$.

Note that if $A \in \mathscr{A}$ and End $(A)$ is a subring of $Q$, then $A \in \mathscr{D}$ and that $A \in \mathscr{C}$ if and only if $A \doteq B$ implies $A \cong B$. The rank one groups are in $\mathscr{C}$ and more generally, any completely decomposable group of finite rank whose type set is a chain in the lattice of types is in $\mathscr{C}$ $[1,9.6]$. Furthemore, the rank two groups in $\mathscr{C}$ have been explicitly computed [2, 9.6]. The groups in $\mathscr{C}$ would appear to have a simpler structure than arbitrary groups in $\mathscr{A}$. For example, if $A \in \mathscr{C}$, then it is immediate from Jónsson's Theorem $[9,2.6]$ that $A$ is indecomposable if and only if $A$ is strongly indecomposable.

\section{Theorem 2. $\mathscr{E}=\mathscr{C} \cap \mathscr{D}$.}

Proof. Let $A \in \mathscr{E}$. If $C$ is any subgroup of $A$ such that $C \supseteqq n A$ for some $n>0$, then $C / n A$ is a subgroup of the cyclic group $A / n A$. It follows that $C / n A=t(A / n A)=t A / n A$ for some divisor $t$ of $n$ and so $C=t A$. In particular, if $B \cong A$ and $A \cong B$, then $A / B$ is finite and so $B=t A$ for some $t>0$, i.e. $A \in \mathscr{D}$. If $A \doteq B$, then $A \supseteqq m B \supseteqq$ $n A$ for some $n, m>0$. Let $C=m B$. Then the above gives $B \cong$ $C=t A \cong A$ for some $t>0$, i.e. $A \in \mathscr{C}$. Thus, $\mathscr{E} \cong \mathscr{C} \cap \mathscr{D}$. On the other hand, let $A \in \mathscr{C} \cap \mathscr{D}$ and suppose $p A \subseteq B \subset A$ for some $p \notin$ $\Pi(A)$. Since $A \in \mathscr{C}, A \cong B$ which together with $A \in \mathscr{D}$ gives $n A=B$ for some $n>0$. Since $n Z_{p} A \subset Z_{p} A, p$ divides $n$ and so $p A=n A=B$, i.e. $A \in \mathscr{E}$.

REMARK 2. Since $\mathscr{E} \subset \mathscr{C}$, Lemma 2 gives an affirmative answer to the conjecture in [2, p. 41]. This has been previously noted by other authors. Let $A$ be a rank two group with a maximal independent set $\left\{x_{1}, x_{2}\right\}$ and $\left(A: x_{1}, x_{2}\right) \rightarrow \Sigma$ be the characteristic in [2, 2.2]. Then a direct computation gives $\Sigma(p)+H^{A}\left(x_{1}\right)(p)+H^{A}\left(x_{2}\right)(p)=\infty$ if and only if $r_{p}(A) \leqq 1$. It follows from [2, Theorem 9.6] that the inde- 
composable rank two groups in $\mathscr{C}$ are precisely the indecomposable rank two groups in $\mathscr{E}$. Finally note that Theorem 2 shows that for $A \in \mathscr{A}$ such that End $(A)$ is a subring of $Q$ (and such groups exist in abundance), $A \in \mathscr{C}$ if and only if $A \in \mathscr{E}$.

Corollary 4. $\mathscr{E}=\{A \in \mathscr{A} \mid A \doteq B$ implies $n A=m B$ for some $n, m>0\}$.

Proof. A modest computation shows that the above set is equal to $\mathscr{C} \cap \mathscr{D}$.

COROLlary 5. The Krull-Schmidt Theorem holds in $\mathscr{E}$.

Proof. Let $A \in \mathscr{E}$ and $A=\bigoplus_{i=1}^{n} A_{i}=\bigoplus_{j=1}^{m} B_{j}$ where $A_{i}$ and $B_{j}$ are indecomposable. Since $\mathscr{E}$ is closed with respect to direct summands and $\mathscr{E} \subseteq \mathscr{C}, A_{i}$ and $B_{j}$ are strongly indecomposable. Jónsson's Theorem gives $n=m$ and for some permutation $\sigma, A_{i} \doteq B_{\sigma(i)}$ and so $A_{i} \cong$ $B_{\sigma(i)}$ for all $i$.

Corollary 6. Let $A$ be a group in $\mathscr{E}$ which is full in V. A is decomposable if and only if there are nonzero $Q$-subspaces $U$ and $W$ of $V$ such that $V=U \oplus W$ and for each $p$, either $U \subseteq d\left(Z_{p} A\right)$ or $W \subseteq d\left(Z_{p} A\right)$.

Proof. Assume $A=B \oplus C$ where $B$ and $C$ are nonzero. Let $U$ and $W$ be the nonzero subspaces of $V$ generated by $B$ and $C$ respectively. Then $V=U \oplus W$. Since $r_{p}(-)$ distributes through direct sums, $B$ is $p$-divisible or $C$ is $p$-divisible. It follows that for each $p$, either $U=Z_{p} B \subseteq d\left(Z_{p} A\right)$ or $W=Z_{p} C \leqq d\left(Z_{p} A\right)$. Conversely, since $U^{(p)} \leqq d\left(Z_{p} A\right)^{(p)} \leqq \delta_{p}(A)$ or $W^{(p)} \subseteq d\left(Z_{p} A\right)^{(p)} \subseteq \delta_{p}(A)$ for each $p$, the modular law gives $\delta_{p}(A)=\left(\delta_{p}(A) \cap U^{(p)}\right) \oplus\left(\delta_{p}(A) \cap W^{(p)}\right)$ for each $p$. It follows from Corollary 3 that $A$ is quasi-decomposable. Since $\mathscr{E} \subseteq$ $\mathscr{C}, A$ is decomposable.

Theorem 3. Let $A$ and $B$ be groups in $\mathscr{E}$ which are full in $V$.

(i) There is an imbedding of $A$ into $B$ if and only if $\tau_{*}(A) \leqq$ $\tau_{*}(B)$ and $\delta(A) \leqq \delta(B)$.

(ii) $A \cong B$ if and only if $\tau_{*}(A)=\tau_{*}(B)$ and $\delta(A) \sim \delta(B)$.

(iii) Let $\tau$ be a type and $\delta \in \mathscr{L}(V)$ be a compatible $Q D$ invariant where $1+r_{Z^{(p)}}\left(\delta_{p}\right) \geqq r_{Q}(V)$ for all $p$. Then there is a $C \in \mathscr{E}$ which is full in $V$ such that $\tau_{*}(C)=\tau$ and $\delta(C)=\delta$.

Proof. This is immediate from Theorems 1 and 2 and Lemma 2. 
REMARK 3. Let $r(A)=1$ and $\delta$ be the $Q D$ invariant associated with a 1 dimensional $V$ such that $\delta_{p}=V^{(p)}$ if $\tau_{*}(A)$ has $\infty$ at $p$ and $\delta_{p}=0$ otherwise. Then $\delta(A)=\delta$. Thus, $\delta(A)$ may be recaptured from $\tau_{*}(A)$ whenever $r(A)=1$. This shows that Theorem 3 generalizes the well-known theorem of Baer [5, 44.2]. In addition, we note that Lemma 8 together with $[5,86.6]$ identifies $\mathscr{E}$ as the class of torsion free Abelian groups which have hereditary generating systems [5, p. 332]. Therefore, we have a solution to the torsion free part of Problem 84 in [5] at least to the extent that our classification of the groups in $\mathscr{E}$ and characterizations of the class $\mathscr{E}$ can be said to determine the structure of the groups in $\mathscr{E}$.

For the remainder of this section we consider the adequacy of our theory for $\mathscr{E}$. For example, Kaplansky in [10] proposed three test problems which any adequate classification of a class of Abelian groups should be able to answer. Let us consider these test problems suitably adjusted to groups $A, B$ and $C$ in $\mathscr{A}$ : ( I ) If $A$ is isomorphic to a subgroup of $B$ and $B$ is isomorphic to a subgroup of $A$, then is $A \cong B$ ?, (II) If $A \oplus A \cong B \oplus B$, then is $A \cong B$ ?, (III) If $A \oplus C \cong B \oplus C$, then is $A \cong B$ ?. All three problems have affirmative answers for $A \in \mathscr{C}$ and therefore, for $A \in \mathscr{E}$. For note that $\mathscr{C}=$ $\{A \in \mathscr{A} \mid(I)$ has an affirmative answer $\}$ and Jónsson's Theorem together with the symmetry and transitivity of the relation of quasi-isomorphism shows (II) and (III) have affirmative answers for $A \in \mathscr{C}$. On the other hand, our theory for $\mathscr{E}$ is only as adequate as our ability to effectively compute with the invariants $\delta(-)$ and $\tau_{*}(-)$. The inner type $\tau_{*}(-)$ is certainly a manageable invariant. The following example shows that the $Q D$ invariants are at least adequate for constructing large families of indecomposables in $\mathscr{E}$ with certain preassigned divisibility properties.

ExAmple 2. Assume $r_{Q}(V)=n>2$. Let $\left\{\alpha_{p}\right\}_{p \in \pi}$ be a sequence of integers where $0 \leqq \alpha_{p} \leqq n$ for all $p, \alpha_{p}<n$ for at least $n+1$ primes $p$ and $\alpha_{q}<n-1$ for at least one prime $q$. Further, let $\tau$ be a type such that $\tau(p)=\infty$ if and only if $\alpha_{p}=n$. Then there is an uncountable family $\left\{A_{i}\right\}_{i \in \lambda}$ such that

(i) $A_{i}$ is an indecomposable group in $\mathscr{E}$ which is full in $V$

(ii) $s_{p}\left(A_{i}\right)=\alpha_{p}$ for all $p$ and $\tau_{*}\left(A_{i}\right)=\tau$

(iii) $A_{i} \not A_{j}$ for $i \neq j$

(iv) if $\alpha_{q}=0$ for some $q$, then $\operatorname{Hom}\left(A_{i}, A_{j}\right)=\{0\}$ for $i \neq j$.

We give the construction in three steps:

I. Let $\left\{U_{p}\right\}_{p \in \pi}$ be a family of $Q$-subspaces of $V$ such that $r_{Q}\left(U_{p}\right)=$ $\alpha_{p}$ for each $p$. Then there is an uncountable subfamily $\left\{\delta^{i}\right\}_{i \in \lambda}$ of $\mathscr{L}(V)$ 
such that for each $i \in \lambda$ and $p \in \pi,\left(\delta^{i}\right)_{p} \cap V=U_{p}, r_{Z^{(p)}}\left(\left(\delta^{i}\right)_{p}\right) \geqq n-1$, and $\delta^{i} \nsim \delta^{j}$ for $i \neq j$.

Proof. If $U_{p}=V$ (i.e. $\alpha_{p}=n$ ), then let $\delta_{p}=V^{(p)}$. For $\alpha_{p}<n$, let $V=U_{p} \oplus U$ and select a pure subgroup $B$ of $Z^{(p)}$ with $r(B)=$ $n-\alpha_{p}$. Imbed $B$ as a full subgroup of $U$. Then $r\left(d\left(B^{(p)}\right)\right)=n-$ $\alpha_{p}-1$ (since $B \in \mathscr{E}$ ) and $d\left(B^{(p)}\right) \cap U=\{0\}$ (since $s_{p}(B)=0$ ). Let $\delta_{p}=$ $U_{p}^{(p)} \oplus d\left(B^{(p)}\right) \subseteq V^{(p)}$. Then $r_{Z^{(p)}}\left(\delta_{p}\right)=n-1$. Since $U_{p} \subseteq \delta_{p}$, the modular law gives $\delta_{p} \cap V=U_{p} \oplus\left(\delta_{p} \cap U\right)=U_{p}$.

Let $q$ be a prime such that $n-\alpha_{q}>1$. Then it is well-known that there is an uncountable family $\left\{B_{i}\right\}_{i \in \rho}$ of pure subgroups of $Z^{(q)}$ where $r\left(B_{i}\right)=n-\alpha_{q}$ and $B_{i} \not B_{j}$ for $i \neq j$. As in the previous paragraph, let $V=U_{q} \oplus U$, imbed $B_{i}$ in $U$, and let $\delta_{q}^{i}=U_{q}^{(q)} \oplus d\left(B_{i}^{(q)}\right)$. We may regard $d\left(B_{i}^{(q)}\right) \in \mathscr{L}_{q}(U)$ and it follows that for $i \neq j, \delta_{q}^{i} \neq \delta_{q}^{j}$ (since if $\delta_{q}^{i}=\delta_{q}^{j}$, then by modularity $\delta\left(B_{i}\right) \sim \delta\left(B_{j}\right)$ and Theorem 3 would give $B_{i} \cong B_{j}$ ).

For $i \in \rho$, define $\delta^{i}$ by $\left(\delta_{i}\right)_{p}=\delta_{p}$ for $p \neq q$ and $\left(\delta^{i}\right)_{q}=\delta_{q}^{i}$. Then $\mathscr{F}=\left\{\delta^{i}\right\}_{i \in \rho}$ is an uncountable family of distinct $Q D$ invariants in $\mathscr{L}(V)$ such that $\left(\delta^{i}\right)_{p} \cap V=U_{p}$ and $r_{Z^{(p)}}\left(\left(\delta^{i}\right)_{p}\right) \geqq n-1$. Since the group of automorphisms of $V$ is countable, the equivalence class determined by a $\delta^{i} \in \mathscr{F} w / r$ to the equivalence relation $\sim$ on $\mathscr{L}(V)$ is countable. It follows that there is an uncountable subset $\lambda \leqq \rho$ such that $\delta^{i} \nsim \delta^{j}$ for $i \neq j$ and $i, j \in \lambda$.

II. Let $\alpha_{1}, \cdots, \alpha_{n+1}$ be integers such that $0 \leqq \alpha_{i}<n$. Then there are $n+1$ subspaces $\left\{V_{i}\right\}_{i=1}^{n+1}$ of $V$ such that:

(a) $r_{Q}\left(V_{i}\right)=\alpha_{i}$, and (b) if $V=U \oplus W$ and for each $i$, either $V_{i} \supseteqq U$ or $V_{i} \supseteqq W$, then $U=\{0\}$ or $W=\{0\}$.

Proof. It is enough to assume $\alpha_{i}=n-1$ in (a) and to construct a family of $n+1$ hyperspaces which satisfy condition (b). Let $X$ be a basis for $V$. Choose $V_{1}, \cdots, V_{n}$ as the hyperspaces generated by the $n$ subsets of $X$ with $n-1$ elements. Let $V_{n+1}$ be any hyperspace which does not interset $X$. Then $\left\{V_{i}\right\}_{i=1}^{n+1}$ satisfies condition (b).

III. We now construct the required family of groups. Since $\alpha_{p}<n$ for at least $n+1$ primes, we may assume that the $n+1$ subspaces of $V$ constructed in (II) are in the family of subspaces $\left\{U_{p}\right\}_{p \in \pi}$ in (I). By hypothesis $\tau$ is compatible with each $\delta^{i}$ in (I). Now use Theorem 3 to obtain a group $A_{i}$ in $\mathscr{E}$ with $\delta\left(A_{j}\right)=\delta^{i}$ and $\tau_{*}\left(A_{i}\right)=\tau$. Since $U_{p}=\left(\delta^{i}\right)_{p} \cap V=d\left(Z_{p} A\right), A_{i}$ is indecomposable [Corollary 6 and (II)] and $s_{p}\left(A_{i}\right)=\alpha_{p}$ [Lemma 2]. Since $\delta^{i} \nsim \delta^{j}$ for $i \neq j, A_{i} \not A_{j}$ for $i \neq j$ [Theorem 3]. Hence, $\left\{A_{i}\right\}_{i \in \lambda}$ is the required family. For part (iv), let $\phi \in \operatorname{Hom}\left(A_{i}, A_{j}\right)$ for $i \neq j$ and assume that $\phi$ is not the zero 
map. Suppose $r_{q}(\operatorname{ker} \phi)=1$, then $r_{q}\left(\phi\left(A_{i}\right)\right)=0$. Since $s_{q}\left(A_{j}\right)=0$, the only $q$-divisible subgroup of $A_{j}$ is $\{0\}$ and so $\phi\left(A_{i}\right)=\{0\}$, a contradiction. Thus, $r_{q}(\operatorname{ker} \phi)=0$ and so ker $\phi=\{0\}$. Since $\phi$ is an imbedding of $A_{i}$ in $A_{j}$, Theorem 3 gives $\delta\left(A_{i}\right) \leqq \delta\left(A_{j}\right)$. Since $s_{p}\left(A_{i}\right)=s_{p}\left(A_{j}\right)$ for all $p$ and $A_{i}, A_{j} \in \mathscr{E}$, it follows that $\delta\left(A_{i}\right) \sim \delta\left(A_{j}\right)$. Since $\tau_{*}\left(A_{i}\right)=\tau_{*}\left(A_{j}\right)$, Theorem 3 gives $A_{i} \cong A_{j}$, a contradiction of (iii). Thus, $\phi$ is the zero map.

In the preceding example the $A_{i}^{\prime} s$ will be purely indecomposable if $\alpha_{p}=0$ for some $p$ [Corollary 6]. On the other hand, if $\alpha_{p}>0$ for all $p$, then the $A_{i}^{\prime} s$ need not be purely indecomposable. In the following example we construct an indecomposable group $A$ in $\mathscr{E}$ which is not purely indecomposable, i.e. $A$ contains a pure, decomposable subgroup.

EXAMPle 3. Assume $r_{Q}(V)=n>2$. Let $q$ and $r$ be distinct primes and $\alpha_{q}, \alpha_{r}$ be positive integers such that $\alpha_{q}+\alpha_{r}<n$. Further, let $\mathscr{F}=\left\{U_{p}\right\}_{p \in \pi}$ be a family of subspaces of $V$ such that:

(i) $r_{Q}\left(U_{q}\right)=\alpha_{q}, r_{Q}\left(U_{r}\right)=\alpha_{r}$ and $U_{r} \cap U_{q}=\{0\}$,

(ii) for all $p, U_{p} \supseteqq U_{q}$ or $U_{p} \supseteqq U_{r}$.

Use the construction in (I) to obtain a group $A$ in $\mathscr{E}$ which is full in $V$ and $\delta_{p}(A) \cap V=U_{p}$ for each $p$. Since $d\left(Z_{p} A\right)=\delta_{p}(A) \cap V, A$ is indecomposable by condition (i) and Corollary 6 . Now let $B$ be the pure hull in $A$ of the subgroups $U_{q} \cap A$ and $U_{r} \cap A$. Then $B$ is a full subgroup of $U_{q} \oplus U_{r}$ and for all $p \in \pi$, either $U_{q} \subseteq d\left(Z_{p} B\right)$ or $U_{r} \leqq d\left(Z_{p} B\right)$. Since $B \in \mathscr{E}$, Corollary 6 shows that $B$ is decomposable. Hence, $A$ is indecomposable but not purely indecomposable.

We briefiy mention another method of constructing uncountable families of indecomposable groups in $\mathscr{E}$ which have the same rank and are pairwise non-isomorphic. Let $\delta \in \mathscr{L}(V)$ such that $1+$ $r_{Z^{(p)}}\left(\delta_{p}\right) \geqq n$ for all $p$ where $n=r_{Q}(V), r_{Z^{(p)}}\left(\delta_{p}\right)<n$ for an infinite number of primes $p$, and $\delta$ does not satisfy part (iii) of Corollary 3. Note that such a $\delta$ exists by (I) and (II) in Example 2. Then there are an uncountable number of distinct types $\left\{\tau_{i}\right\}_{i \in \lambda}$ which are compatible with $\delta$. Now apply Theorem 3 to obtain for each $i$, a rank $n$ group $A_{i}$ in $\mathscr{E}$ with $\tau_{*}\left(A_{i}\right)=\tau_{i}$ and $\delta\left(A_{i}\right)=\delta$. The resulting $A_{2}^{\prime} s$ are pairwise non-isomorphic [Theorem 3] and indecomposable [Corollary 3].

\section{The endomorphisms of groups in $\mathscr{E}$.}

Lemma 9. Let $A$ be a reduced group in $\mathscr{E}$ and $A=\bigoplus_{i=1}^{n} A_{i}$ where $A_{i}$ is indecomposable. Then End $(A) \cong \bigoplus_{i=1}^{n}$ End $\left(A_{i}\right)$.

Proof. It is sufficient to show Hom $\left(A_{i}, A_{j}\right)=\{0\}$ for $i \neq j$. Let 
$\phi \in \operatorname{Hom}\left(A_{i}, A_{j}\right)$. If $A_{i}$ is $p$-divisible, then so is $\phi\left(A_{i}\right)$. Thus, $Z_{p} \phi\left(A_{i}\right) \cong$ $d\left(Z_{p} A_{j}\right)$ whenever $r_{p}\left(A_{i}\right)=0$. On the other hand, $Z_{p} \phi\left(A_{i}\right) \subseteq Z_{p} A_{j}=$ $d\left(Z_{p} A_{j}\right)$ whenever $r_{p}\left(A_{j}\right)=0$. Since $A \in \mathscr{E}$, for each $p, r_{p}\left(A_{i}\right)=0$ or $r_{p}\left(A_{j}\right)=0$. It follows that $\phi\left(A_{i}\right)=\bigcap_{p \in \Pi} Z_{p} \phi\left(A_{i}\right) \subseteq \bigcap_{p \in I} d\left(Z_{p} A_{j}\right)=\{0\}$ (since $A_{j}$ is reduced). Hence, $\dot{\phi}$ is the zero map.

THEOREM 4. If $A$ is an indecomposable group in $\mathscr{E}$, then every endomorphism of $A$ is an integral multiple of an automorphism of $A$.

REMARK. The author is indebted to the referee for suggesting the following proof, which is considerably more natural than the original one.

Proof. If $\phi \in$ End $(A)$ is $1-1$, then Theorem 2 gives $\phi(A)=n A$ for some $0 \neq n \in Z$ and so $(1 / n) \phi$ is an automorphism of $A$. Therefore, it is sufficient to show that nonzero endomorphisms of $A$ are monomorphisms. Suppose not. Then choose a nonzero singular endomorphism $\phi$ such that $r(\phi(A))$ is minimal. Let $B=\phi(A)$. Then $B$ is clearly indecomposable and by induction on $r(A),\left.\phi\right|_{B}$ is a multiple of an automorphism of $B$. Hence, $\phi(B)=n B$ for some $n>0$ and so $\operatorname{ker} \phi \cap B=\{0\}$. Let $n \alpha \in n A$. Then $\phi(n \alpha) \in n B=\phi(B)$ and so $n \alpha \in$ ker $\phi \oplus B$. Thus, $A \doteq B \oplus \operatorname{ker} \phi$ but $A$ is indecomposable and in $\mathscr{C}$, a contradication.

We remark that a theorem of P. Griffith's [7] says that a pure subgroup $B$ of the $Z$-adic completion of $Z$ is purely indecomposable if and only if the nonzero endomorphisms of pure subgroups of $B$ are monomorphisms. For finite rank $B$, this result is immediate from Theorem 4. Another immediate consequence of Theorem 4 is that the characteristic and fully invariant subgroups of an indecomposable group in $\mathscr{E}$ coincide.

Before stating some other consequences of Theorem 4, we recall some elementary facts about the $Z$-adic completions of reduced groups in $\mathscr{E}$. If $R$ is a ring with identity, then we denote the additive subgroup of $R$ by $R^{+}$and require that subrings contain the identity of $R$. Let $A$ be a reduced group in $\mathscr{E}$. Then the $Z$-adic completion of $A$, denoted by $\hat{A}$, is $\Pi Z^{(p)}$ where $p$ runs over $\Pi \backslash \Pi(A)$ [8]. In addition, $\hat{A}$ is a commutative ring with identity under component-wise multiplication. For $\alpha \in \hat{A}$, let $l_{\alpha}$ denote the left-multiplication by $\alpha$. It is well-known that an endomorphism of $\hat{A}^{+}$is a $l_{\alpha}$ for some $\alpha \in \hat{A}$. The automorphisms of $\hat{A}^{+}$correspond to the left multiplications by units. Regard $A$ as a pure dense subgroup of $\hat{A}$. Since $\hat{A}^{+}$is pure injective [6], every endomorphism (automorphism) of $A$ uniquely extends to an endomorphism (automorphism) of $\hat{A}^{+}$. In particular, 
the identity map on $A$ extends to $l_{1}$. It follows that End $(A)$ is isomorphic to a subring of $\hat{A}$. Finally, if $R$ is a pure subring of $\hat{A}$, then $\Pi\left(R^{+}\right)=\Pi(A)$ and End $\left(R^{+}\right) \cong R$ (since $1 \in R$ ).

CoRollary 7. If $A$ is a reduced group in $\mathscr{E}$, then End $(A)$ is a ring direct sum of Principal Ideal Domains. In particular, if $A$ is indecomposable, then End $(A)$ is a Principal Ideal Domain which is isomorphic to a pure subring of $\hat{A}$ and $Q \otimes$ End $(A)$ is an algebraic number field.

Proof. In view of Lemma 9 it is enough to assume that $A$ is a reduced, indecomposable group in $\mathscr{E}$. Regard $A$ as embedded in $\hat{A}$ as a pure dense subgroup and so by the previous remarks End $(A)$ may be regarded as a subring $R$ of $\hat{A}$. In this setting Theorem 4 says that the elements of $R$ are integral multiples of units in $\hat{A}$. Thus, $R$ is a domain and the purity of $A$ in $\hat{A}$ shows that $\hat{A} / R$ is torsion free as a group, i.e. $R$ is a pure subring of $A$. Let $I$ be a nonzero ideal in $R$ and $W=\left\{n \in Z \mid n>0\right.$ and $\left.l_{n} \in I\right\} . \quad W \neq \Phi$ by Theorem 4. Let $m=\min W$ and $\lambda \in I$. Then it follows from a routine use of the division algorithm that $\lambda=l_{m} \phi$ for some $\phi \in R$. Thus, $R$ is a Principal Ideal Domain. That $Q \otimes$ End $(A)$ is an algebraic number field is immediate from Theorem 4 and the finiteness of the rank of $\operatorname{Hom}(A, A)$.

Corollary 8. If $A$ is a reduced, indecomposable group in $\mathscr{E}$, then $r(A)=r(\operatorname{Hom}(A, A)) r_{R}(A)$ where $R=$ End $(A)$.

Proof. $A$ is a torsion free module over the Principal Ideal Domain $R$. Let $x_{1}, \cdots x_{n}$ be a maximal $R$-independent set in $A$ where $n=r_{R}(A)$. Then $I=\bigoplus_{i=1}^{n} R x_{i}$ is a full free $R$-submodule of $A$ and so by Theorem $4, I$ as a group is full in $A$. This gives the equation.

Before stating the final two corollaries to Theorem 4, we recall two definitions from [12]. $A$ is called strongly homogeneous if given two pure rank one subgroups $B$ and $C$, there is an automorphism $\phi$ of $A$ such that $\phi(B)=C$. A is called a special group if it is a strongly homogeneous $Q D$ group in $\mathscr{E}$. All rank one groups are trivially strongly homogeneous and the rank one special groups are precisely those with non-nil type. We will need the following property of special groups [12]: If $A$ is a special group, then $A \cong \operatorname{Hom}(A, A)$.

Corollary 9. If $A$ is a reduced group in $\mathscr{E}$, then $\operatorname{Hom}(A, A)$ is a direct sum of special groups. In particular, if $A$ is indecomposable, then $\operatorname{Hom}(A, A)$ is a special group. 
Proof. By Lemma 9 it is enough to assume that $A$ is indecomposable. Regard End $(A)$ as a pure subring of $\hat{A}$. Let $C$ be the pure hull in $\operatorname{Hom}(A, A)$ of the identity and $B$ be any rank one pure subgroup of Hom $(A, A)$. $B$ contains a unit $b$ by Theorem 4 and so $l_{b}$ is an automorphism of $\operatorname{Hom}(A, A)$. Since $l_{b}(C) \cap B$ is a nonzero pure subgroup of both $l_{b}(C)$ and $B, l_{b}(C)=B$. Furthermore, Hom $(A, A)$ is a $Q D$ group (since it is homogeneous of the type of $C$, which is nonnil). Thus, $\operatorname{Hom}(A, A)$ is a special group.

The previous corollaries are combined in the following in order to survey the finite rank pure subrings of the $Z$-adic completions of groups in $\mathscr{E}$.

CoROLlaRY 10. Let $\Pi^{\prime} \subseteq \Pi$ and $R$ be a finite rank pure subring of $T\left(\Pi^{\prime}\right)=\Pi Z^{(p)}$ where $p$ runs over $\Pi^{\prime}$. Then:

(i) $R$ is a ring direct sum of Principal Ideal Domains

(ii) $R^{+}$is a direct sum of special groups and every group, which is a direct sum of special groups and whose Z-adic completion is $T\left(\Pi^{\prime}\right)$, is the additive subgroup of some $R$.

(iii) In particular, if $R$ is a Principal Ideal Domain, then $R^{+}$ is special and every special group, whose Z-adic completion is $T\left(\Pi^{\prime}\right)$, is the additive subgroup of some Principal Ideal Domain $R$.

Proof. These are all immediate from Corollaries 7 and 9 together with the facts that End $\left(R^{+}\right) \cong R$, Hom $\left(R^{+}, R^{+}\right) \cong R^{+}$and for $A$ special, $\operatorname{Hom}(A, A) \cong A$.

The following shows that the relationship between a strongly homogeneous group in $\mathscr{E}$ and its endomorphism ring is the same as that between a rank one group and its endomorphism ring.

THEOREM 5. Let $A$ be an indecomposable group in $\mathscr{E}$ and $I$ a full free subgroup of $A$. Then the following are equivalent:

(i) $A$ is strongly homogeneous

(ii) $Q D(A, I)$ is a special group which is isomorphic to $\operatorname{Hom}(A, A)$

(iii) $A$ is a torsion free, rank one End $(A)$-module.

Proof. Assume (i). Let $J$ be a full free subgroup of $A$ such that $Q D(A, J)$ is a characteristic subgroup of $A$ [Lemma 6]. Since $Q D(A, I) \cong Q D(A, J)$, it is enough to show $Q D(A, J)$ is a special group isomorphic to $\operatorname{Hom}(A, A)$. Let $B$ and $C$ be pure rank one subgroups of $Q D(A, J)$. Then there is an automorphism $\phi^{\prime}$ of $A$ such that $\phi^{\prime}(P H(B))=P H(C)$ where $P H(-)$ denotes the pure hull in $A$. Let $\dot{\phi}$ be the restriction of $\phi^{\prime}$ to $Q D(A, J)$. Then $\phi$ is an automor- 
phism of $Q D(A, J)$. It follows from the purity of $B$ and $C$ in $Q D(A, J)$ that $P H(B) \cap Q D(A, J)=B$ and $P H(C) \cap Q D(A, J)=C$. Thus, $\phi(B)=$ $C$ and so $Q D(A, J)$ is special. This together with Corollary 2 gives $Q D(A, J) \cong \operatorname{Hom}(A, A)$, which is (ii). Assume (ii). Then $r(A)=$ $r(\operatorname{Hom}(A, A))$ and (iii) follows from Corollary 8. Assume (iii). Let $B$ and $C$ be pure rank one subgroups of $A$ and $0 \neq x \in B, 0 \neq y \in C$. Then there are nonzero $\phi^{\prime}, \lambda^{\prime} \in$ End $(A)$ such that $\phi^{\prime}(x)=\lambda^{\prime}(y)$. By Theorem $4 \lambda^{\prime}=n \lambda$ and $\phi^{\prime}=m \phi$ for some automorphisms $\phi$ and $\lambda$ and some nonzero $n, m$. Note that $B=P H((m x))$ and $C=P H((n y))$. Let $\theta=\lambda^{-1} \phi$. Since $\theta$ is an automorphism of $A, \theta(B)$ is a pure rank 1 subgroup of $A$ which contains ny. Thus, $C \cong \theta(B)$ and it follows from purity that $C=\theta(B)$, which is (i).

Corollary 11. If $A$ is an indecomposable group in $\mathscr{E}$ of prime rank, then $A$ is either strongly homogenous or End $(A)$ is a subring of $Q$.

Proof. Immediate from Corollary 8 and Theorem 5.

Corollary 12. Let $A$ be a reduced group in $\mathscr{E}$. Then $r(A)=$ $r(\operatorname{Hom}(A, A))$ if and only if $A$ is a direct sum of strongly homogeneous groups.

Proof. Immediate from Lemma 9 and Theorem 5.

We follow Reid [11] in calling $A$ irreducible if $A$ has no nontrivial pure, fully invariant subgroups. Since strongly homogeneous groups in $\mathscr{A}$ have no nontrivial pure, characteristic subgroups, strongly homogeneous groups are always irreducible.

Corollary 13. Let $A \in \mathscr{E}$. Then $A$ is irreducible if and only if $A$ is strongly homogeneous.

Proof. We need only check the "only if" and may assume that $A$ is reduced. Since $A$ is an irreducible group in $\mathscr{E}, A$ is indecomposable. Therefore, $A$ is a torsion free module over its endomorphism ring $R$. Let $0 \neq x \in A$. Then $R x$ is a nonzero fully invariant subgroup of $A$ and is full in $P H(R x)$. It follows that $P H(R x)$ is a nonzero pure, fully invariant subgroup of $A$. Thus, $P H(R x)=A$ which implies $r(A)=r(R)$ and so $A$ is strongly homogeneous [Corollary 12]. 


\section{REFERENCES}

1. R. A. Beaumont and R. S. Pierce, Torsion-free rings, III. Jour. Math., 5 (1961), 61-98.

2. - Torsion free groups of rank 2, Mem. Amer. Math. Soc., 38 (1961).

3. - Quasi-Isomorphism of p-Groups, in Proceedings of the Colloquium on Abelian groups (Tihany), Akademia Kiado, Budapest (1964), 13-27.

4. D. W. Dubois, Cohesive groups and p-adic integers, Publ. Math. Debrecen 12 (1965), 51-58.

5. L. Fuchs, Abelian Groups, Hungarian Academy of Sciences, Budapest (1958).

6. - Infinite Abelian Groups, Vol. 1, Academic Press, N. Y. (1970).

7. P. Griffith, Purely indecomposable torsion-free groups, Proc. Amer. Math. Soc., 18 (1967), 738-742.

8. D. K. Harrison, Infinite Abelian groups and homological methods, Ann. of Math., 69 (1959), 366-391.

9. B. Jónsson, On direct decompositions of torsion free Abelian groups, Math. Scand., 7 (1959), 361-371.

10. I. Kaplansky, Infinite Abelian Groups, University of Michigan Press, Ann Arbor, 1954.

11. J. D. Reid, On the ring of quasi-endomorphisms of a torsion-free Abelian group, in Topics in Abelian groups, Scott, Foresman and Co., Chicago (1963), 51-68.

12. F. Richman, A Class of Rank-2-Torsion Free Groups, in Proceedings of the Colloquium on Abelian groups (Montpellier), Donod, Paris (1968), 327-334.

13. R. B. Warfield, Jr., Homomorphisms and duality for torsion-free groups, Math. Zeitschr., 107 (1968), 189-200.

Recieved December 21, 1970 and in revised form October 28, 1971.

UNIVERSITY OF VICTORIA 



\section{PACIFIC JOURNAL OF MATHEMATICS}

\section{EDITORS}

\section{H. SAMELSON}

Stanford University

Stanford, California 94305

\section{R. HOBBY}

University of Washington Seattle, Washington 98105

\section{J. DugundJI}

Department of Mathematics University of Southern California Los Angeles, California 90007

RICHARD ARENS

University of California Los Angeles, California 90024

\section{ASSOCIATE EDITORS}
E. F. BECKENBACH
B. H. Neumann
F. WoLF
K. YoSHIDA

\section{SUPPORTING INSTITUTIONS}

UNIVERSITY OF BRITISH COLUMBIA CALIFORNIA INSTITUTE OF TECHNOLOGY

UNIVERSITY OF CALIFORNIA

MONTANA STATE UNIVERSITY

UNIVERSITY OF NEVADA

NEW MEXICO STATE UNIVERSITY

OREGON STATE UNIVERSITY

UNIVERSITY OF OREGON

OSAKA UNIVERSITY

\author{
UNIVERSITY OF SOUTHERN CALIFORNIA \\ STANFORD UNIVERSITY \\ UNIVERSITY OF TOKYO \\ UNIVERSITY OF UTAH \\ WASHINGTON STATE UNIVERSITY \\ UNIVERSITY OF WASHINGTON \\ * * * * \\ AMERICAN MATHEMATICAL SOCIETY \\ NAVAL WEAPONS CENTER
}

The Supporting Institutions listed above contribute to the cost of publication of this Journal, but they are not owners or publishers and have no responsibility for its content or policies.

Mathematical papers intended for publication in the Pacific Journal of Mathematics should be in typed form or offset-reproduced, (not dittoed), double spaced with large margins. Underline Greek letters in red, German in green, and script in blue. The first paragraph or two must be capable of being used separately as a synopsis of the entire paper. The editorial "we" must not be used in the synopsis, and items of the bibliography should not be cited there unless absolutely necessary, in which case they must be identified by author and Journal, rather than by item number. Manuscripts, in duplicate if possible, may be sent to any one of the four editors. Please classify according to the scheme of Math. Rev. Index. to Vol. 39. All other communications to the editors should be addressed to the managing editor, Richard Arens, University of California, Los Angeles, California, 90024.

50 reprints are provided free for each article; additional copies may be obtained at cost in multiples of 50 .

The Pacific Journal of Mathematics is published monthly. Effective with Volume 16 the price per volume (3 numbers) is $\$ 8.00$; single issues, $\$ 3.00$. Special price for current issues to individual faculty members of supporting institutions and to individual members of the American Mathematical Society: $\$ 4.00$ per volume; single issues $\$ 1.50$. Back numbers are available.

Subscriptions, orders for back numbers, and changes of address should be sent to Pacific Journal of Mathematics, 103 Highland Boulevard, Berkeley, California, 94708.

PUBLISHED BY PACIFIC JOURNAL OF MATHEMATICS, A NON-PROFIT CORPORATION

Printed at Kokusai Bunken Insatsusha (International Academic Printing Co., Ltd.), 270, 3-chome Totsuka-cho, Shinjuku-ku, Tokyo 160, Japan. 


\section{Pacific Journal of Mathematics}

\section{Vol. 40, No. $3 \quad$ November, 1972}

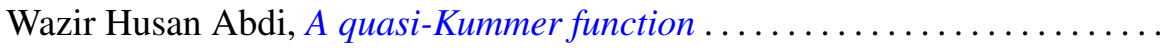

Vasily Cateforis, Minimal injective cogenerators for the class of modules of

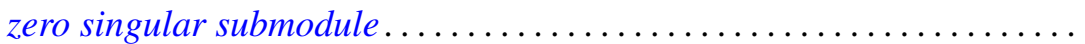

W. Wistar (William) Comfort and Anthony Wood Hager, Cardinality of

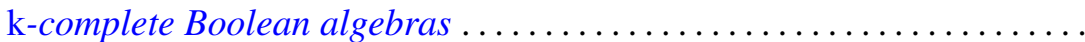

Richard Brian Darst and Gene Allen DeBoth, Norm convergence of martingales of Radon-Nikodym derivatives given a $\sigma$-lattice ..........

M. Edelstein and Anthony Charles Thompson, Some results on nearest points and support properties of convex sets in $c_{0} \ldots \ldots \ldots \ldots \ldots$

Richard Goodrick, Two bridge knots are alternating knots .

Jean-Pierre Gossez and Enrique José Lami Dozo, Some geometric properties related to the fixed point theory for nonexpansive mappings ..........

Dang Xuan Hong, Covering relations among lattice varieties .............

Carl Groos Jockusch, Jr. and Robert Irving Soare, Degrees of members of $\Pi_{1}^{0}$

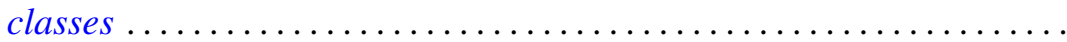

565

575

605

Leroy Milton Kelly and R. Rottenberg, Simple points in pseudoline

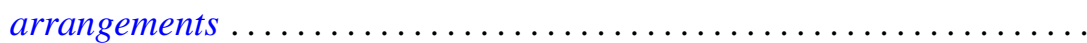

Joe Eckley Kirk, Jr., The uniformizing function for a class of Riemann surfaces....

Glenn Richard Luecke, Operators satisfying condition $\left(G_{1}\right)$ locally ... 629

T. S. Motzkin, On L $(S)$-tuples and l-pairs of matrices ... . .

Charles Estep Murley, The classification of certain classes of torsion free

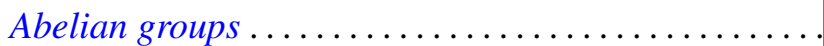

Louis D. Nel, Lattices of lower semi-continuous functions and associated topological spaces.

David Emroy Penney, II, Establishing isomorphism between tame prime

knots in $E^{3}$. . .

Daniel Rider, Functions which operate on $\mathscr{F} L_{p}(T), 1<p<2$

Thomas Stephen Shores, Injective modules over duo rings ...

Stephen Simons, A convergence theorem with boundary. .

703

Stephen Simons, Maximinimax, minimax, and antiminimax theorems and a

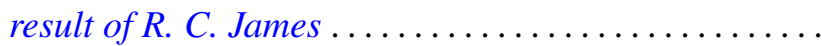

Stephen Simons, On Ptak's combinatorial lemma ........

Stuart A. Steinberg, Finitely-valued $f$-modules............

Pui-kei Wong, Integral inequalities of Wirtinger-type and fourth-order

elliptic differential inequalities .

Yen-Yi Wu, Completions of Boolean algebras with partially additive

operators ..................................

Phillip Lee Zenor, On spaces with regular $G_{\delta}$-diagonals . . . 